Bulletin of Electrical Engineering and Informatics

Vol. 10, No. 6, December 2021, pp. 3274 3281

ISSN: 2302-9285, DOI: 10.11591/eei.v10i6.2981

\title{
Exact secure outage probability performance of uplink- downlink multiple access network under imperfect CSI
}

Dinh-Thuan Do, Minh-Sang Van Nguyen

Department of Electronics and Communications, Faculty of Electronics Technology, Industrial University of Ho Chi Minh City (IUH), Vietnam

\begin{tabular}{|c|c|}
\hline Art & BSTRACT \\
\hline Arti & \multirow{8}{*}{$\begin{array}{l}\text { In this paper, we study uplink-downlink non-orthogonal multiple access (NOMA) sys- } \\
\text { tems by considering the secure performance at the physical layer. In the considered } \\
\text { system model, the base station acts a relay to allow two users at the left side commu- } \\
\text { nicate with two users at the right side. By considering imperfect channel state infor- } \\
\text { mation (CSI), the secure performance need be studied since an eavesdropper wants to } \\
\text { overhear signals processed at the downlink. To provide secure performance metric, } \\
\text { we derive exact expressions of secrecy outage probability (SOP) and and evaluating } \\
\text { the impacts of main parameters on SOP metric. The important finding is that we can } \\
\text { achieve the higher secrecy performance at high signal to noise ratio (SNR). Moreover, } \\
\text { the numerical results demonstrate that the SOP tends to a constant at high SNR. Fi- } \\
\text { nally, our results show that the power allocation factors, target rates are main factors } \\
\text { affecting to the secrecy performance of considered uplink-downlink NOMA systems. }\end{array}$} \\
\hline $\operatorname{Rec}$ & \\
\hline Revised Aug 6, 2021 & \\
\hline & \\
\hline Keyn & \\
\hline Channel st & \\
\hline Non-orthogonal multiple & \\
\hline & \\
\hline
\end{tabular}

This is an open access article under the CC BY-SA license.

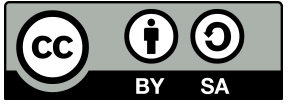

\section{Corresponding Author:}

Dinh-Thuan Do

Department of Electronics and Communications, Faculty of Electronics Technology

Industrial University of Ho Chi Minh City (IUH)

Ho Chi Minh City 700000, Vietnam

Email: dodinhthuan@iuh.edu.vn

\section{INTRODUCTION}

Due to high demands in terms of system capacity and spectrum efficiency, the traditional orthogonal multiple access (OMA) has been unable to meet the user needs associated with the rapid growth of internet of things (IoT) and mobile communications [1]-[7]. In order to meet the heavy demand for mobile services, non-orthogonal multiple access (NOMA) is researched in recent years with promising applications [8], [9]. In some scenarios, NOMA benefits to device-to-device communications [10], [11] and cognitive radio (CR)aided NOMA [12]-[14] and these are considered as potential key technologies for the fifth generation mobile communications (5G). The authors Do, et al. in [13] studied the secondary network of the considered CRNOMA by enabling the relaying scheme. In such network, the secondary transmitter is able to conduct energy harvesting $(\mathrm{EH})$ to perform signal forwarding to distant secondary users. Two main metrics including outage behavior and throughput performance are studied in the context of EH-assisted CR-NOMA while imperfect successive interference cancellation (SIC) is considered. Reference Do, et al. [14] presented relay-aided CRNOMA networks to improve the performance of far users by enabling partial relay selection architecture. They explored system performance in terms of full-duplex (FD) and half duplex (HD) relays for both uplink and downlink communications.

Recently, an alternative approach is enabled to conduct cryptography at physical layer security (PLS) 
has considered. This method is more advanced due to complications of secure techniques applied at higher layers in existing RFID systems. To aim to decrease chance of eavesdroppers getting information from the legal transmitter, the wireless channel characteristics is utilized to PLS-based system act relevant approach to against eavesdroppers' overhearing operations. The authors in [15]-[20] studied PLS applied for a 5G NOMA system. The authors in [15] explored the two-user case and then extend our results to a multi-user case. The main results indicated that the given users' data rate corresponds to positive secrecy rate. The PLS of millimeter wave (mmWave) NOMA networks was studied for mmWave channels in [16] by examining imperfect CSI at receivers and the limited scattering characteristics of concerned channels. the formula of the secrecy outage probability (SOP) was derived since the system adopts random distributions of legitimate users and eavesdroppers. While [15], [16] presented NOMA downlink scenario, the authors in [18] investigated uplink secure NOMA system. The typical system including one base station, one eavesdropper and multiple users. However, there is lack of work considering secure performance of uplink-downlink NOMA system under imperfect CSI circumstance, which motive us to study secure outage probability in this article.

\section{SYSTEM MODEL}

In this system model, we consider uplink-downlink of two pairs of source-destination $S_{1}-D_{1}, S_{2}-D_{2}$ under existence of eavesdropper $E$, shown in Figure 1. The flat slow Rayleigh fading is assumed for all links and the channel coefficients pertaining to the links $S_{1} \rightarrow R, S_{2} \rightarrow R, R \rightarrow D_{1}, R \rightarrow D_{2}$ and $R \rightarrow E$ are denoted as $g_{1 r}, g_{2 r}, g_{d 1}, g_{d 2}$ and $g_{e}$, respectively. Accordingly, the corresponding channel power gains conform to $\left|g_{1 r}\right|^{2} \sim C N\left(0, \lambda_{1 r}\right),\left|g_{2 r}\right|^{2} \sim C N\left(0, \lambda_{2 r}\right),\left|g_{d 1}\right|^{2} \sim C N\left(0, \lambda_{d 1}\right),\left|g_{d 2}\right|^{2} \sim C N\left(0, \lambda_{d 2}\right)$ and $\left|g_{e}\right|^{2} \sim C N\left(0, \lambda_{e}\right)$, respectively.

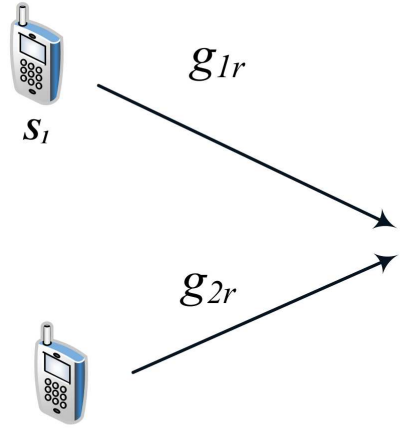

$S_{2}$
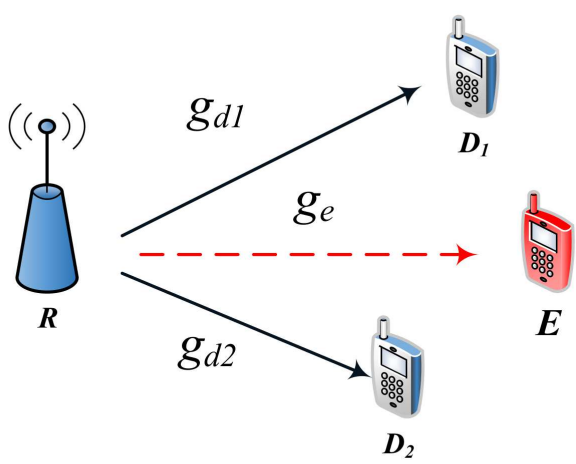

$\boldsymbol{E}$

Figure 1. System model

Two sources sends their signals to the relay $R$ in the same time. In particular, the received signal at $R$ can be expressed as [21].

$$
y_{S-R}=\left(g_{1 r}+h_{1 r}\right) \sqrt{a_{1} P_{s 1}} z_{1}+\left(g_{2 r}+h_{2 r}\right) \sqrt{a_{2} P_{s 2}} z_{2}+n_{r},
$$

where $P_{s i}$ represents the transmit power at $S_{i} ; n_{r}$ is denoted as the variance of the additive white Gaussian noise (AWGN) at $R$ with $n_{r} \sim C N\left(0, N_{0}\right) ; z_{i}$ is the signal of $D_{i} ; a_{i}$ are the power allocation coefficients of $z_{i}$ transmitted signals with $a_{1}+a_{2}=1$ and assuming that $a_{1}>a_{2} ; h_{i r}$ is the error term related to imperfect CSI, which follow a complex Gaussian distributed random variable with $C N\left(0, \sigma_{h i}^{2}\right)$. In this circumstance, $\sigma_{h i}^{2}$ is assumed as constant [22].

With regard to higher priority, the relay always first decodes $z_{1}$ by considering $z_{2}$ as noise. Following NOMA principle, the system can performs SIC to decode $z_{2}$. To further compute system performance metric, we first calculaye the received signal-to-interference-plus-noise ratio (SINR) for symbol $z_{1}$. Then, we can 
determine the signal-to-noise (SNR) for symbol $z_{2}$. In particular, these values are given as,

$$
\gamma_{z_{1}}^{u}=\frac{a_{1} \rho_{s 1}\left|g_{1 r}\right|^{2}}{a_{2} \rho_{s 2}\left|g_{2 r}\right|^{2}+a_{1} \rho_{s 1} \sigma_{h 1}^{2}+a_{2} \rho_{s 2} \sigma_{h 2}^{2}+1}, \gamma_{z_{2}}^{u}=\frac{a_{2} \rho_{s 2}\left|g_{2 r}\right|^{2}}{a_{1} \rho_{s 1} \sigma_{h 1}^{2}+a_{2} \rho_{s 2} \sigma_{h 2}^{2}+1},
$$

where $\rho_{s 1}=\frac{P_{s 1}}{N_{0}}, \rho_{s 2}=\frac{P_{s 2}}{N_{0}}$ whoch represent SNR at sources.

Next, the signals received at the destinations can be determined. The relay in the second phase wants to send superimposed signal generated from $z_{i}$ to destinations $D_{i}$. Therefore, we can achieve the received signal at $D_{i}$ as.

$$
y_{R-D_{i}}=\left(g_{d i}+h_{d i}\right)\left(\sqrt{a_{1} P_{r}} z_{1}+\sqrt{a_{2} P_{r}} z_{2}\right)+n_{d i}
$$

where $h_{d i}$ is the error term which is considered as a complex Gaussian distributed random variable with $C N\left(0, \sigma_{d i}^{2}\right) ; n_{d i}$ stand for the variance of the additive white Gaussian noise (AWGN) at $D_{i}$ with $n_{d i} \sim$ $C N\left(0, N_{0}\right)$.

By processing signals transferred from $R, D_{1}$ decodes its intended symbol $z_{1}$ when it treats $z_{2}$ as noise. Then, we compute corresponding SINR of $z_{1}$ at $D_{1}$ as.

$$
\gamma_{z_{1}}^{d}=\frac{a_{1} \rho_{r}\left|g_{d 1}\right|^{2}}{a_{2} \rho_{r}\left|g_{d 1}\right|^{2}+\rho_{r} \sigma_{d 1}^{2}+1}
$$

where $\rho_{r}=\frac{P_{r}}{N_{0}}$.

At the other side, user $D_{2}$ first decodes $z_{1}$ and then employing SIC to achieve signal $z_{2}$. At user $D_{2}$, SINR of $z_{1}$ and the SNR of $z_{2}$ at are expressed respectively by.

$$
\gamma_{1 \rightarrow 2}^{d}=\frac{a_{1} \rho_{r}\left|g_{d 2}\right|^{2}}{a_{2} \rho_{r}\left|g_{d 2}\right|^{2}+\rho_{r} \sigma_{d 2}^{2}+1}, \gamma_{z_{2}}^{d}=\frac{a_{2} \rho_{r}\left|g_{d 2}\right|^{2}}{\rho_{r} \sigma_{d 2}^{2}+1}
$$

The received signal at $E$ from $R$ can be expressed as.

$$
y_{E}=g_{e}\left(\sqrt{a_{1} P_{r}} z_{1}+\sqrt{a_{2} P_{r}} z_{2}\right)+n_{e}
$$

After employing the parallel interference cancellation (PIC) scheme, the received SINR at the eavesdropper to detect $D_{i}$ 's message can be formulated by [23].

$$
\gamma_{z_{i}}^{e}=a_{i} \rho_{e}\left|g_{e}\right|^{2}
$$

where $\rho_{e}=\frac{P_{r}}{n_{e}}$.

In the next step, the achievable secrecy rates of two pairs of users can be examined. Following (2), (4), and (7), we compute the achievable secrecy rates of $S_{1}-D_{1}$ as.

$$
\chi_{1}=\frac{1}{2}\left[\log _{2} \min \left(\frac{1+\gamma_{z_{1}}^{u}}{1+\gamma_{z_{1}}^{e}}, \frac{1+\gamma_{z_{1}}^{d}}{1+\gamma_{z_{1}}^{e}}\right)\right]^{+}
$$

where $[x]^{+}=\max \{0, x\}$.

From (2), (5) and (7), the achievable secrecy rates of $S_{2}-D_{2}$ is written as.

$$
\chi_{2}=\frac{1}{2}\left[\log _{2} \min \left(\frac{1+\gamma_{z_{2}}^{u}}{1+\gamma_{z_{2}}^{e}}, \frac{1+\gamma_{1 \rightarrow 2}^{d}}{1+\gamma_{z_{2}}^{e}}, \frac{1+\gamma_{z_{2}}^{d}}{1+\gamma_{z_{2}}^{e}}\right)\right]^{+}
$$

In the next section, we intends to examine secure performance metric which relies on secrecy rates obtained in these steps. 


\section{SECRECY OUTAGE PROBABILITY (SOP)}

\subsection{SOP for user pair $S_{1}-D_{1}$}

To evaluate SOP performance, the secrecy outage event $S_{1}-D_{1}$ need be known when $z_{1}$ cannot be securely decoded by $R$ or by $D_{1}$, the SOP for $S_{1}-D_{1}$ can be expressed as [23], [24].

$$
\begin{aligned}
& S O P_{D_{1}}^{S_{1}}=\operatorname{Pr}\left(\chi_{1}<R_{1}\right) \\
& =1-\operatorname{Pr}\left(\min \left(\frac{1+\gamma_{z_{1}}^{u}}{1+\gamma_{z_{1}}}, \frac{1+\gamma_{z_{1}}^{d}}{1+\gamma_{z_{1}}}\right) \geq \gamma_{d_{1}}\right) \\
& =1-\underbrace{\operatorname{Pr}\left(\frac{1+\gamma_{z_{1}}^{u}}{1+\gamma_{z_{1}}^{e}} \geq \gamma_{d_{1}}\right)}_{\Psi_{1}} \underbrace{\operatorname{Pr}\left(\frac{1+\gamma_{z_{1}}^{d}}{1+\gamma_{z_{1}}^{e}} \geq \gamma_{d_{1}}\right)}_{\Psi_{2}} .
\end{aligned}
$$

\subsubsection{Proposition 1}

The SOP of user pair $S_{1}-D_{1}$ is approximated computed as.

$$
S O P_{D_{1}}^{S_{1}}=1+\frac{\rho_{s 1} \lambda_{1 r} \eta_{1}}{\gamma_{d_{1}} \rho_{e} \lambda_{e} \lambda_{e} a_{2} \rho_{s 2} \lambda_{2 r}} \exp \left(\beta_{1} \beta_{2}-\frac{\phi_{1} \kappa_{1}}{a_{1} \rho_{s 1} \lambda_{1 r}}\right) \operatorname{Ei}\left(-\beta_{1} \beta_{2}\right),
$$

where $\phi_{i}=\gamma_{d_{i}}-1, \gamma_{d_{i}}=2^{2 R_{i}},(i=1,2), R_{i}$ is the target data rate for user $D_{i}, \kappa_{1}=a_{1} \rho_{s 1} \sigma_{h 1}^{2}+a_{2} \rho_{s 2} \sigma_{h 2}^{2}+1$, $\kappa_{2}=\rho_{r} \sigma_{d 1}^{2}+1, \eta_{1}=\int_{0}^{\infty} \exp \left(-\frac{\kappa_{2} \gamma_{d_{1}} a_{1} \rho_{e} x+\phi_{1} \kappa_{2}}{\left(a_{1}-a_{2} \phi_{1}-a_{1} a_{2} \gamma_{d_{1}} \rho_{e} x\right) \rho_{r} \lambda_{d 1}}-\frac{x}{\lambda_{e}}\right) d x, \beta_{1}=\frac{\gamma_{d_{1}} \rho_{e} \lambda_{e} \kappa_{1}+\rho_{s 1} \lambda_{1 r}}{\gamma_{d_{1}} \rho_{e} \lambda_{e} a_{2} \rho_{s 2}}, \beta_{2}=$ $\frac{\phi_{1} a_{2} \rho_{s 2}}{a_{1} \rho_{s 1} \lambda_{1 r}}+\frac{1}{\lambda_{2 r}}$.

\subsubsection{Proof}

From (10), $\Psi_{1}$ can written by.

$$
\begin{aligned}
& \Psi_{1}=\operatorname{Pr}\left(\frac{1+\gamma_{z_{1}}^{u}}{1+\gamma_{z_{1}}^{u}} \geq \gamma_{d_{1}}\right) \\
& =\operatorname{Pr}\left(\gamma_{z_{1}}^{u} \geq \phi_{1}+\gamma_{d_{1}} \gamma_{z_{1}}^{e}\right) \\
& =\operatorname{Pr}\left(\left|g_{1 r}\right|^{2} \geq \frac{\phi_{1} a_{2} \rho_{s 2}\left|g_{2 r}\right|^{2}+\phi_{1} \kappa_{1}+\gamma_{d_{1}} a_{1} \rho_{e}\left|g_{e}\right|^{2}\left(a_{2} \rho_{s 2}\left|g_{2 r}\right|^{2}+\kappa_{1}\right)}{a_{1} \rho_{s 1}}\right) \\
& =\int_{0}^{\infty} \int_{0}^{\infty}\left(1-F_{\left|g_{1 r}\right|^{2}}\left(\frac{\gamma_{d_{1}} a_{1} \rho_{e} x\left(a_{2} \rho_{s 2} y+\kappa_{1}\right)+\phi_{1} a_{2} \rho_{s 2} y+\phi_{1} \kappa_{1}}{a_{1} \rho_{s 1}}\right)\right) f_{\left|g_{e}\right|^{2}}(x) f_{\left|g_{2 r}\right|^{2}}(y) d x d y
\end{aligned}
$$

where $\phi_{1}=\gamma_{d_{1}}-1$ and $\kappa_{1}=a_{1} \rho_{s 1} \sigma_{h 1}^{2}+a_{2} \rho_{s 2} \sigma_{h 2}^{2}+1$. By conveying the Rayleigh distribution with probability density function (PDF) and cumulative density function (CDF) $f_{|X|^{2}}(x)=\frac{1}{\varphi_{X}} \exp \left(-\frac{x}{\varphi_{X}}\right), F_{|X|^{2}}(x)=$ $1-\exp \left(-\frac{x}{\varphi_{X}}\right), \Psi_{1}$ can be formulated by

$$
\begin{aligned}
& \Psi_{1}=\int_{0}^{\infty} \int_{0}^{\infty} \exp \left(-\frac{\gamma_{d_{1}} a_{1} \rho_{e} x\left(a_{2} \rho_{s 2} y+\kappa_{1}\right)+\phi_{1} a_{2} \rho_{s 2} y+\phi_{1} \kappa_{1}}{a_{1} \rho_{s 1} \lambda_{1 r}}\right) \frac{1}{\lambda_{e}} \exp \left(-\frac{x}{\lambda_{e}}\right) \frac{1}{\lambda_{2 r}} \exp \left(-\frac{y}{\lambda_{2 r}}\right) d x d y \\
& =\frac{1}{\lambda_{e}} \frac{1}{\lambda_{2 r}} \exp \left(-\frac{\phi_{1} \kappa_{1}}{a_{1} \rho_{s 1} \lambda_{1 r}}\right) \int_{0}^{\infty} \int_{0}^{\infty} \exp \left(-\left(\frac{\gamma_{d_{1}} \rho_{e}\left(a_{2} \rho_{s 2} y+\kappa_{1}\right)}{\rho_{s 1} \lambda_{1 r}}+\frac{1}{\lambda_{e}}\right) x\right) \exp \left(-\left(\frac{\phi_{1} a_{2} \rho_{s 2}}{a_{1} \rho_{s 1} \lambda_{1 r}}+\frac{1}{\lambda_{2 r}}\right) y\right) \\
& \times d x d y \\
& =\frac{1}{\lambda_{2 r}} \exp \left(-\frac{\phi_{1} \kappa_{1}}{a_{1} \rho_{s 1} \lambda_{1 r}}\right) \int_{0}^{\infty} \frac{\rho_{s 1} \lambda_{1 r}}{\gamma_{d_{1} \rho_{e} \lambda_{e} a_{2} \rho_{s 2} y+\gamma_{d_{1}} \rho_{e} \lambda_{e} \kappa_{1}+\rho_{s 1} \lambda_{1 r}} \exp \left(-\left(\frac{\phi_{1} a_{2} \rho_{s 2}}{a_{1} \rho_{s 1} \lambda_{1 r}}+\frac{1}{\lambda_{2 r}}\right) y\right) d x d y .}
\end{aligned}
$$
as.

By applying some polynomial expansion manipulations and based on [25] (3.352.4) a5, we obtain $\Psi_{1}$

$$
\Psi_{1}=-\frac{\rho_{s 1} \lambda_{1 r}}{\gamma_{d_{1}} \rho_{e} \lambda_{e} a_{2} \rho_{s 2} \lambda_{2 r}} \exp \left(\beta_{1} \beta_{2}-\frac{\phi_{1} \kappa_{1}}{a_{1} \rho_{s 1} \lambda_{1 r}}\right) \operatorname{Ei}\left(-\beta_{1} \beta_{2}\right),
$$

where $\beta_{1}=\frac{\gamma_{d_{1}} \rho_{e} \lambda_{e} \kappa_{1}+\rho_{s 1} \lambda_{1 r}}{\gamma_{d_{1}} \rho_{e} \lambda_{e} a_{2} \rho_{s 2}}, \beta_{2}=\frac{\phi_{1} a_{2} \rho_{s 2}}{a_{1} \rho_{s 1} \lambda_{1 r}}+\frac{1}{\lambda_{2 r}}$. 
From (10), $\Psi_{2}$ can written by

$$
\begin{aligned}
& \Psi_{2}=\operatorname{Pr}\left(\frac{1+\gamma_{z_{1}}^{d}}{1+\gamma_{z_{1}}^{e}} \geq \gamma_{d_{1}}\right) \\
& =\operatorname{Pr}\left(\gamma_{z_{1}}^{d} \geq \phi_{1}+\gamma_{d_{1}} \gamma_{z_{1}}^{e}\right) \\
& =\operatorname{Pr}\left(\left|g_{d 1}\right|^{2} \geq \frac{\kappa_{2} \gamma_{d_{1}} a_{1} \rho_{e}\left|g_{e}\right|^{2}+\phi_{1} \kappa_{2}}{\left(a_{1}-a_{2}\left(\phi_{1}+\gamma_{d_{1}} a_{1} \rho_{e}\left|g_{e}\right|^{2}\right)\right) \rho_{r}}\right) \\
& =\int_{0}^{\infty}\left(1-F_{\left|g_{d 1}\right|^{2}}\left(\frac{\kappa_{2} \gamma_{d_{1}} a_{1} \rho_{e} x+\phi_{1} \kappa_{2}}{\left(a_{1}-a_{2}\left(\phi_{1}+\gamma_{d_{1}} a_{1} \rho_{e} x\right)\right) \rho_{r}}\right)\right) f_{\left|g_{e}\right|^{2}}(x) d x \\
& =\int_{0}^{\infty} \exp \left(-\frac{\kappa_{2} \gamma_{d_{1}} a_{1} \rho_{e} x+\phi_{1} \kappa_{2}}{\left(a_{1}-a_{2} \phi_{1}-a_{1} a_{2} \gamma_{d_{1}} \rho_{e} x\right) \rho_{r} \lambda_{d 1}}\right) \frac{1}{\lambda_{e}} \exp \left(-\frac{x}{\lambda_{e}}\right) d x \\
& =\frac{1}{\lambda_{e}} \int_{0}^{\infty} \exp \left(-\frac{\kappa_{2} \gamma_{d_{1}} a_{1} \rho_{e} x+\phi_{1} \kappa_{2}}{\left(a_{1}-a_{2} \phi_{1}-a_{1} a_{2} \gamma_{d_{1}} \rho_{e} x\right) \rho_{r} \lambda_{d 1}}-\frac{x}{\lambda_{e}}\right) d x,
\end{aligned}
$$

where $\kappa_{2}=\rho_{r} \sigma_{d 1}^{2}+1$.

It completes the proof.

\subsection{SOP for $S_{2}-D_{2}$}

Similar the user pair $S_{1}-D_{1}$, we need examine the secrecy outage event for user pair $S_{2}-D_{2}$. Several cases are examined such as $R$ cannot detect $z_{2}, D_{2}$ cannot detect its own message $z_{2}$ when $D_{1}$ can detect $z_{1}$ successfully. As a result, we compute the SOP for user pair $S_{2}-D_{2}$ as.

$$
\begin{aligned}
& S O P_{D_{2}}^{S_{2}}=\operatorname{Pr}\left(\chi_{2}<R_{2}\right) \\
& =1-\operatorname{Pr}\left(\min \left(\frac{1+\gamma_{z_{2}}^{u}}{1+\gamma_{z_{2}}^{e}}, \frac{1+\gamma_{1 \rightarrow 2}^{d}}{1+\gamma_{z_{2}}^{e}}, \frac{1+\gamma_{z_{2}}^{d}}{1+\gamma_{z_{2}}^{e}}\right) \geq \gamma_{d_{2}}\right) \\
& =1-\underbrace{\operatorname{Pr}\left(\frac{1+\gamma_{z_{2}}^{u}}{1+\gamma_{z_{2}}^{e}} \geq \gamma_{d_{2}}\right)}_{\Phi_{1}} \underbrace{\operatorname{Pr}\left(\frac{1+\gamma_{1 \rightarrow 2}^{d}}{1+\gamma_{z_{2}}^{e}} \geq \gamma_{d_{2}}\right)}_{\Phi_{2}} \underbrace{\operatorname{Pr}\left(\frac{1+\gamma_{z_{2}}^{d}}{1+\gamma_{z_{2}}^{e}} \geq \gamma_{d_{2}}\right)}_{\Phi_{3}} .
\end{aligned}
$$

\subsubsection{Proposition 2}

The exact SOP for user pair $S_{2}-D_{2}$ is calculated by.

$$
S O P_{D_{2}}^{S_{2}}=1-\frac{a_{2} \rho_{s 2} \lambda_{2 r}}{\kappa_{1} \gamma_{d_{2}} a_{2} \rho_{e} \lambda_{e}+a_{2} \rho_{s 2} \lambda_{2 r}} \frac{a_{2} \rho_{r} \lambda_{d 2} \eta_{2}}{\left(\kappa_{3} \gamma_{d_{2}} a_{2} \rho_{e} \lambda_{e}+a_{2} \rho_{r} \lambda_{d 2}\right) \lambda_{e}} \exp \left(-\frac{\kappa_{1} \phi_{2}}{a_{2} \rho_{s 2} \lambda_{2 r}}-\frac{\kappa_{3} \phi_{2}}{a_{2} \rho_{r} \lambda_{d 2}}\right)
$$

where $\kappa_{3}=\rho_{r} \sigma_{d 2}^{2}+1, \eta_{2}=\int_{0}^{\infty} \exp \left(-\frac{\kappa_{3}\left(\phi_{2}+\gamma_{d_{2}} a_{2} \rho_{e} x\right)}{\left(a_{1}-a_{2} \phi_{2}-\gamma_{d_{2}} a_{2} a_{2} \rho_{e} x\right) \rho_{r} \lambda_{d 2}}-\frac{x}{\lambda_{e}}\right) d x$.

\subsubsection{Proof}

From (16), $\Phi_{1}$ can be calculated as.

$$
\begin{aligned}
& \Phi_{1}=\operatorname{Pr}\left(\frac{1+\gamma_{z_{2}}^{u}}{1+\gamma_{z_{2}}^{e}} \geq \gamma_{d_{2}}\right) \\
& =\operatorname{Pr}\left(\gamma_{z_{2}}^{u} \geq \phi_{2}+\gamma_{d_{2}} \gamma_{z_{2}}^{e}\right) \\
& =\operatorname{Pr}\left(\left|g_{2 r}\right|^{2} \geq \frac{\kappa_{1} \phi_{2}+\kappa_{1} \gamma_{d_{2}} a_{2} \rho_{e}\left|g_{e}\right|^{2}}{a_{2} \rho_{s 2}}\right) \\
& =\int_{0}^{\infty}\left(1-F_{\left|g_{2 r}\right|^{2}}\left(\frac{\kappa_{1} \phi_{2}+\kappa_{1} \gamma_{d_{2}} a_{2} \rho_{e} x}{a_{2} \rho_{s 2}}\right)\right) f_{\left|g_{e}\right|^{2}}(x) d x \\
& =\frac{1}{\lambda_{e}} \exp \left(-\frac{\kappa_{1} \phi_{2}}{a_{2} \rho_{s 2} \lambda_{2 r}}\right) \int_{0}^{\infty} \exp \left(-\left(\frac{\kappa_{1} \gamma_{d_{2}} a_{2} \rho_{e}}{a_{2} \rho_{s 2} \lambda_{2 r}}+\frac{1}{\lambda_{e}}\right) x\right) d x \\
& =\frac{a_{2} \rho_{s 2} \lambda_{2 r}}{\kappa_{1} \gamma_{d_{2}} a_{2} \rho_{e} \lambda_{e}+a_{2} \rho_{s 2} \lambda_{2 r}} \exp \left(-\frac{\kappa_{1} \phi_{2}}{a_{2} \rho_{s 2} \lambda_{2 r}}\right)
\end{aligned}
$$

where $\phi_{2}=\gamma_{d_{2}}-1$. 
Next, $\Phi_{2}$ can be computed as.

$$
\begin{aligned}
& \Phi_{2}=\operatorname{Pr}\left(\frac{1+\gamma_{1 \rightarrow 2}^{d}}{1+\gamma_{z_{2}}^{e}} \geq \gamma_{d_{2}}\right) \\
& =\operatorname{Pr}\left(\gamma_{1 \rightarrow 2}^{d} \geq \phi_{2}+\gamma_{d_{2}} \gamma_{z_{2}}^{e}\right) \\
& =\operatorname{Pr}\left(\left|g_{d 2}\right|^{2} \geq \frac{\kappa_{3}\left(\phi_{2}+\gamma_{d_{2}} a_{2} \rho_{e}\left|g_{e}\right|^{2}\right)}{\left(a_{1}-a_{2} \phi_{2}-\gamma_{d_{2}} a_{2} a_{2} \rho_{e}\left|g_{e}\right|^{2}\right) \rho_{r}}\right) \\
& =\int_{0}^{\infty}\left(1-F_{\left|g_{d 2}\right|^{2}}\left(\frac{\kappa_{3}\left(\phi_{2}+\gamma_{d_{2}} a_{2} \rho_{e} x\right)}{\left(a_{1}-a_{2} \phi_{2}-\gamma_{d_{2}} a_{2} a_{2} \rho_{e} x\right) \rho_{r}}\right)\right) f_{\left|g_{e}\right|^{2}}(x) d x \\
& =\frac{1}{\lambda_{e}} \int_{0}^{\infty} \exp \left(-\frac{\kappa_{3}\left(\phi_{2}+\gamma_{d_{2}} a_{2} \rho_{e} x\right)}{\left(a_{1}-a_{2} \phi_{2}-\gamma_{d_{2}} a_{2} a_{2} \rho_{e} x\right) \rho_{r} \lambda_{d 2}}-\frac{x}{\lambda_{e}}\right) d x,
\end{aligned}
$$

where $\kappa_{3}=\rho_{r} \sigma_{d 2}^{2}+1$.

Using result from (16), $\Phi_{3}$ is expressed by.

$$
\begin{aligned}
& \Phi_{3}=\operatorname{Pr}\left(\frac{1+\gamma_{z_{2}}^{d}}{1+\gamma_{z_{2}}^{e}} \geq \gamma_{d_{2}}\right) \\
& =\operatorname{Pr}\left(\gamma_{z_{2}}^{d} \geq \phi_{2}+\gamma_{d_{2}} \gamma_{z_{2}}^{e}\right) \\
& =\operatorname{Pr}\left(\left|g_{d 2}\right|^{2} \geq \frac{\kappa_{3} \phi_{2}+\kappa_{3} \gamma_{d_{2}} a_{2} \rho_{e}\left|g_{e}\right|^{2}}{a_{2} \rho_{r}}\right) \\
& =\int_{0}^{\infty}\left(1-F_{\left|g_{d 2}\right|^{2}}\left(\frac{\kappa_{3} \phi_{2}+\kappa_{3} \gamma_{d_{2}} a_{2} \rho_{e} x}{a_{2} \rho_{r}}\right)\right) f_{\left|g_{e}\right|^{2}}(x) d x \\
& =\frac{1}{\lambda_{e}} \exp \left(-\frac{\kappa_{3} \phi_{2}}{a_{2} \rho_{r} \lambda_{d 2}}\right) \int_{0}^{\infty} \exp \left(-\left(\frac{\kappa_{3} \gamma_{d_{2}} a_{2} \rho_{e}}{a_{2} \rho_{r} \lambda_{d 2}}+\frac{1}{\lambda_{e}}\right) x\right) d x \\
& =\frac{a_{2} \rho_{r} \lambda_{d 2}}{\kappa_{3} \gamma_{d_{2}} a_{2} \rho_{e} \lambda_{e}+a_{2} \rho_{r} \lambda_{d 2}} \exp \left(-\frac{\kappa_{3} \phi_{2}}{a_{2} \rho_{r} \lambda_{d 2}}\right)
\end{aligned}
$$

This is end of the proof.

\section{SIMULATION RESULTS}

To conduct these simulations, we set $\rho=\rho_{s 1}=\rho_{s 2}=\rho_{r}, \sigma=\sigma_{h 1}^{2}=\sigma_{h 2}^{2}=\sigma_{d 1}^{2}=\sigma_{d 2}^{2}$.

In Figure 2, we show the SOP versus transmit SNR at the source. It can be seen clearly that higher transmit power at the source will enhance SOP performance, especially in high SNR region. By assigning different power allocation factors, the second user pair $S_{2}-D_{2}$ outperforms that that of $S_{1}-D_{1}$ when SNR is greater than $20 \mathrm{~dB}$. The higher power factor $a_{1}=0.9$ leads to improvement of SOP for $S_{1}-D_{1}$. Similarly, we evaluate the impact of rates $R_{1}<R_{2}$ on SOP performance, shown in Figure 3. The lower requirement of target rates indicate the best SOP among three cases of $R_{1}<R_{2}$ examined. In Figure 4, we can see similar SOP performance for two user pairs when we change $\sigma$. It can be concluded that the quality of channels make influence on SOP metric..

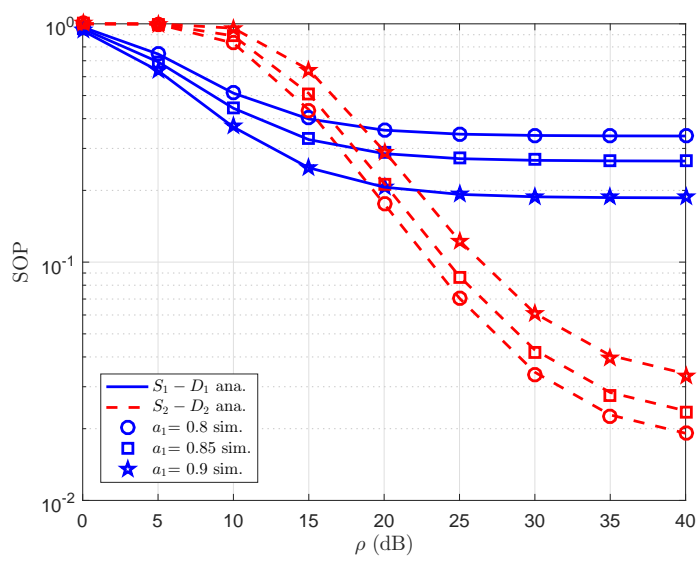

Figure 2. SOP for $S_{1}-D_{1}$ and $S_{2}-D_{2}$ versus $\rho$ as changing $a_{1}$ with $R_{1}=R_{2}=1(\mathrm{bps} / \mathrm{Hz}), \sigma=0.001$ $\lambda_{1 r}=\lambda_{2 r}=1, \lambda_{d 2}=2, \lambda_{e}=1, \rho_{e}=-20(\mathrm{~dB})$

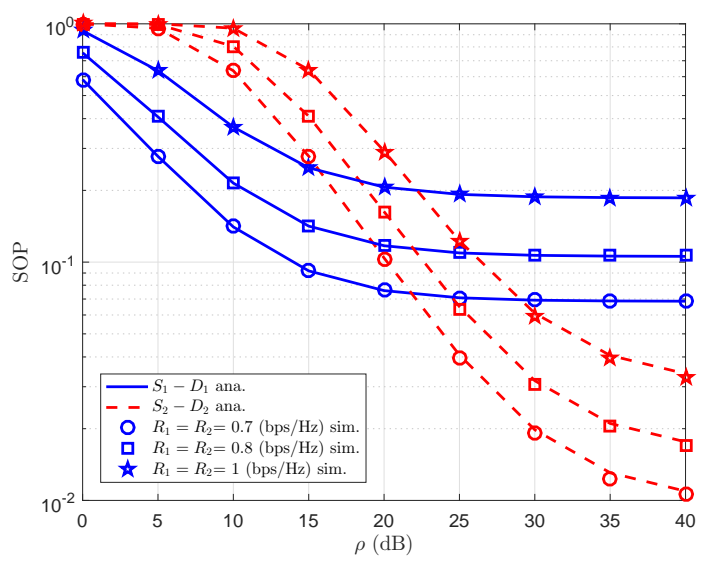

Figure 3. SOP for $S_{1}-D_{1}$ and $S_{2}-D_{2}$ versus $\rho$ as changing $R_{1}=R_{2}$ with $a_{1}=0.9, \sigma=0.001$, $\lambda_{1 r}=\lambda_{2 r}=1, \lambda_{d 2}=2, \lambda_{e}=1, \rho_{e}=-20(\mathrm{~dB})$ 


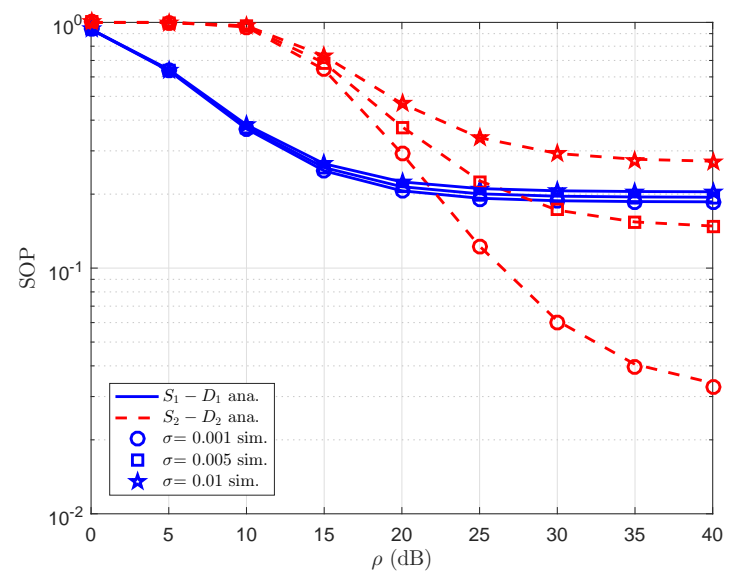

Figure 4. SOP for $S_{1}-D_{1}$ and $S_{2}-D_{2}$ versus $\rho$ as changing $\sigma$ with $a_{1}=0.9, R_{1}=R_{2}=1$ (bps/Hz), $\lambda_{1 r}=\lambda_{2 r}=1, \lambda_{d 2}=2, \lambda_{e}=1, \rho_{e}=-20(\mathrm{~dB})$

\section{CONCLUSION}

This paper investigates the joint uplink and downlink approach to evaluate SOP performance of two user pairs. By assigning fixed power allocation, we can derive exact formulas of SOP for two user pairs. Specifically, we can conclude that SOP will be enhanced at high transmit power at the sources. We further examine the impacts of target rate on SOP performance. Under the existence of eavesdropper, we guarabtee operation of uplink-downlink if we control the quality of channels. Furthermore, we have found that the imperfect CSI has slight impact on SOP performance.

\section{REFERENCES}

[1] H. Huang, W. Xia, J. Xiong, J. Yang, G. Zheng and X. Zhu, "Unsupervised learning-based fast beamforming design for downlink MIMO," in IEEE Access, vvol. 7, pp. 7599-7605, 2019, doi: 10.1109/ACCESS.2018.2887308.

[2] G. Gui, H. Sari and E. Biglieri, "A new definition of fairness for non-orthogonal multiple access," in IEEE Communications Letters, vol. 23, no. 7, pp. 1267-1271, July 2019, doi: 10.1109/LCOMM.2019.2916398.

[3] B. Wang, F. Gao, S. Jin, H. Lin and G. Y. Li, "Spatial-and frequencywideband effects in millimeter-wave massive MIMO systems," in IEEE Transactions on Signal Processing, vol. 66, no. 13, pp. 3393-3406, 1 July, 2018, doi: 10.1109/TSP.2018.2831628.

[4] H. Xie, F. Gao, S. Zhang and S. Jin, "A unified transmission strategy for TDD/FDD massive MIMO systems with spatial basis expansion model," in IEEE Transactions on Vehicular Technology, vol. 66, no. 4, pp. 3170-3184, April 2017, doi: 10.1109/TVT.2016.2594706.

[5] Z. M. Fadlullah, et al., "State-of-the-art deep learning: Evolving machine intelligence toward tomorrow's intelligent network traffic control systems," in IEEE Communications Surveys \& Tutorials, vol. 19, no. 4, pp. 2432-2455, Fourthquarter 2017, doi: 10.1109/COMST.2017.2707140.

[6] N. Kato, et al., "The deep learning vision for heterogeneous network traffic control: Proposal, challenges, and future perspective," in IEEE Wireless Communications, vol. 24, no. 3, pp. 146-153, June 2017, doi: 10.1109/MWC.2016.1600317WC.

[7] F. Tang, B. Mao, Z. M. Fadlullah and N. Kato, "On a novel deep-learningbased intelligent partially overlapping channel assignment in SDN-IoT," in IEEE Communications Magazine, vol. 56, no. 9, pp. 80-86, Sept. 2018, doi: 10.1109/MCOM.2018.1701227.

[8] Dinh-Thuan Do and Anh-Tu Le, "NOMA based cognitive relaying: Transceiver hardware impairments, relay selection policies and outage performance comparison," Computer Communications, vol. 146, pp. 144-154, October 2019, doi: 10.1016/j.comcom.2019.07.023.

[9] X. Li, Q. Wang, Y. Liu, T. A. Tsiftsis, Z. Ding and A. Nallanathan, "UAV-Aided Multi-Way NOMA Networks with Residual Hardware Impairments," in IEEE Wireless Communications Letters, vol. 9, no. 9, pp. 1538-1542, September 2020, doi: 10.1109/LWC.2020.2996782.

[10] X. Li, et al., "Cooperative Wireless-Powered NOMA Relaying for B5G IoT Networks with Hardware Impairments and Channel Estimation Errors," in IEEE Internet of Things Journal, vol. 8, no. 7, pp. 5453-5467, April 2021, doi: 10.1109/JIOT.2020.3029754. 
[11] Anh-Tu Le and Dinh-Thuan Do, "Implement of multiple access technique by wireless power transfer and relaying network," Bulletin of Electrical Engineering and Informatics, vol. 10, no. 2, pp. 793-800, April 2021, doi: 10.11591/eei.v10i2.1903.

[12] Thi-Anh Hoang, Chi-Bao Le and Dinh-Thuan Do, "Security performance analysis for power domain NOMA employing in cognitive radio networks," Bulletin of Electrical Engineering and Informatics, vol. 9, no. 3, pp. 1046-1054, June 2020, doi: 10.11591/eei.v9i3.1639.

[13] Dinh-Thuan Do, A. Le and B. M. Lee, "NOMA in Cooperative Underlay Cognitive Radio Networks Under Imperfect SIC," in IEEE Access, vol. 8, pp. 86180-86195, 2020, doi: 10.1109/ACCESS.2020.2992660.

[14] Dinh-Thuan Do, M. V. Nguyen, F. Jameel, R. Jäntti and I. S. Ansari, "Performance Evaluation of Relay-Aided CR-NOMA for Beyond 5G Communications," in IEEE Access, vol. 8, pp. 134838-134855, 2020, doi: 10.1109/ACCESS.2020.3010842.

[15] C. Zhang, F. Jia, Z. Zhang, J. Ge and F. Gong, "Physical Layer Security Designs for 5G NOMA Systems With a Stronger Near-End Internal Eavesdropper," in IEEE Transactions on Vehicular Technology, vol. 69, no. 11, pp. 13005-13017, November 2020, doi: 10.1109/TVT.2020.3018234.

[16] S. Huang, M. Xiao and H. V. Poor, "On the Physical Layer Security of Millimeter Wave NOMA Networks," in IEEE Transactions on Vehicular Technology, vol. 69, no. 10, pp. 11697-11711, October 2020, doi: 10.1109/TVT.2020.3017086.

[17] R. M. Christopher and D. K. Borah, "Physical Layer Security for Weak User in MISO NOMA Using Directional Modulation (NOMAD)," in IEEE Communications Letters, vol. 24, no. 5, pp. 956-960, May 2020, doi: 10.1109/LCOMM.2020.2975193.

[18] K. Cao, B. Wang, H. Ding, L. Lv, J. Tian and F. Gong, ”On the Security Enhancement of Uplink NOMA Systems With Jammer Selection," in IEEE Transactions on Communications, vol. 68, no. 9, pp. 5747-5763, September 2020, doi: 10.1109/TCOMM.2020.3003665.

[19] H. -M. Wang and X. Zhang, "UAV Secure Downlink NOMA Transmissions: A Secure Users Oriented Perspective," in IEEE Transactions on Communications, vol. 68, no. 9, pp. 5732-5746, September 2020, doi: 10.1109/TCOMM.2020.3002268.

[20] B. Li, X. Qi, K. Huang, Z. Fei, F. Zhou and R. Q. Hu, "Security-Reliability Tradeoff Analysis for Cooperative NOMA in Cognitive Radio Networks," in IEEE Transactions on Communications, vol. 67, no. 1, pp. 83-96, January 2019, doi: 10.1109/TCOMM.2018.2873690.

[21] S. Arzykulov, T. A. Tsiftsis, G. Nauryzbayev and M. Abdallah, "Outage Performance of Cooperative Underlay CRNOMA With Imperfect CSI," in IEEE Communications Letters, vol. 23, no. 1, pp. 176-179, January 2019, doi: 10.1109/LCOMM.2018.2878730.

[22] Z. Yang, Z. Ding, P. Fan and G. K. Karagiannidis, "On the Performance of Non-orthogonal Multiple Access Systems With Partial Channel Information," in IEEE Transactions on Communications, vol. 64, no. 2, pp. 654-667, February 2016, doi: 10.1109/TCOMM.2015.2511078.

[23] J. Chen, L. Yang and M. Alouini, "Physical Layer Security for Cooperative NOMA Systems," in IEEE Transactions on Vehicular Technology, vol. 67, no. 5, pp. 4645-4649, May 2018, doi: 10.1109/TVT.2017.2789223.

[24] Y. Feng, S. Yan, C. Liu, Z. Yang and N. Yang, "Two-Stage Relay Selection for Enhancing Physical Layer Security in Non-Orthogonal Multiple Access," in IEEE Transactions on Information Forensics and Security, vol. 14, no. 6, pp. 1670-1683, June 2019, doi: 10.1109/TIFS.2018.2883273.

[25] I. S. Gradshteyn and I. M. Ryzhik, "Table of Integrals, Series and Products, 6th ed," Academic Press, New York, NY, USA, 2000. 